\title{
The Characteristic of Overhang Object to Material Usage on FDM 3D Printing Technology
}

\author{
Redyarsa Dharma Bintara ${ }^{1 *}$, Aminnudin ${ }^{1}$, Zumrotul $_{\text {Ida }^{2}}$, Ferian Rizki Arbianto ${ }^{1}$, \\ Dani Prasetiyo ${ }^{1}$ \\ ${ }^{1}$ Mechanical Engineering Depatment, State University of Malang, Jl. Semarang 5 Malang, Malang, \\ 65145, Indonesia \\ ${ }^{2}$ Mechanical Engineering Department, National Central University, No. 300, Zhongda Rd., Zhongli \\ District, Taoyuan City 32001, Taiwan (R.O.C.) \\ *Corresponding author:redyarsa.dharma.ft@um.ac.id
}

\begin{abstract}
Fuse Deposition Modeling (FDM) 3D printing is one of additive manufacturing technology which physical 3D model is build up layer by layer. The support structure is almost involved on the process if overhang shape is met on the 3D model. It has main function to prevent the 3D printed model from collapsing. Commonly, the single material source of FDM 3D printer machine is to supply building two structure, structure of main 3D object and support structure. Hence, our goal optimizes the using of support material for reducing the main material usage. Furthermore, the sixteen of variation overhang angle is set to the 3D model. All models are printed into two kind of 3D printed model, printed model with support structure addition and without support addition. The weight of each 3D printed model is measured by weight scale with accuracy of tool is $10^{-4} \mathrm{~g}$. Then the quality and the weight of 3D printed model are compared and analyzed. The result shows that the average overweight of 3D printed model with support structure addition is $40.41 \%$ than without support structure addition. Furthermore, there are several the $3 \mathrm{D}$ printed models without support structure that fail printed on variety model with $0^{\circ}$ until $11^{\circ}$ of overhang angle. The conclusion of this study is that the support structure can prevent the $3 \mathrm{D}$ printed model from collapsing but it does not need be built up if the overhang angle more than $11^{\circ}$.

Copyright @ 2019. Journal of Mechanical Engineering Science and Technology

All rights reserved
\end{abstract}

Keyword: 3D Printing, overhang, support structure

\section{Introduction}

The manufacture process is commonly involving several tools to produce a part. Additive manufacturing (AM) is a technology that can be used to improve quality of manufacturing process. The AM is also known as 3D printing technology which can be utilized to build a physical 3D model by depositing material layer by layer. The main application of 3D printing technology is for imitating a 3D model. Using imitation model, a user can evaluate and optimize the 3D model for developing a desire physical 3D model, especially for mass production. Several 3D printer machines has launched in the market such as Selective Laser Sintering (SLS), Direct Metal Laser Sintering (DMLS), Fused Deposition Modeling (FDM), and Stereolithography (SLA) [1]. All of it is differenced by type of material used for construct 3D printed model. Currently, FDM 3D printing disposes to be used because it has relative small machine and cheap of material price. The 3D printing technology has applied in several fields such training and education [2], pre-operative planning bone surgery [3], automotive [4], and 
industrial application [5]. Hence, a user should optimize the material used for reducing cost production [6].

One method to reduce the cost production is optimizing support structure material usage. The support structure is built for prevent the main object from collapsing. It is generated when the overhang shape is met. Commonly, the 3D printer machine, especially FDM 3D printer uses single source of material for printing the main 3D model and support structure. Hence, the support structure should be optimized for reducing the material usage. Furthermore, several researchers have suggested that the minimum selfsupporting angle for DMLS printed model is 45 degrees through experimental testing [7]. Other researcher also conducts the overhang shape for developing the model of support structure [8]. In this study, the overhang angle is investigated to find out the optimize support structure and get the best 3D printed model setting.

\section{Methods}

The support structure is an important shape to be built up when the overhang shape is met. The main task of support structure is to prevent the main 3D object deposit layer from collapsing or falling. In this study, the 3D model is drawn into CAD software with sixteen of varied overhang angle $(\theta)$ is varied $0^{\circ}$ up to $15^{\circ}$ as depict on the Figure 1 . Furthermore, the 3D model is exported into ".stl" extension file before imported into 3D printer software. In the 3D printer software, the stereolithography (STL) file of 3D model is sliced for simulating the path of deposited material sequence. Then the path information of deposit material can be exported into ".gcode" extension file and it is used by $3 \mathrm{D}$ printer machine as reference path to deposit the melting material.

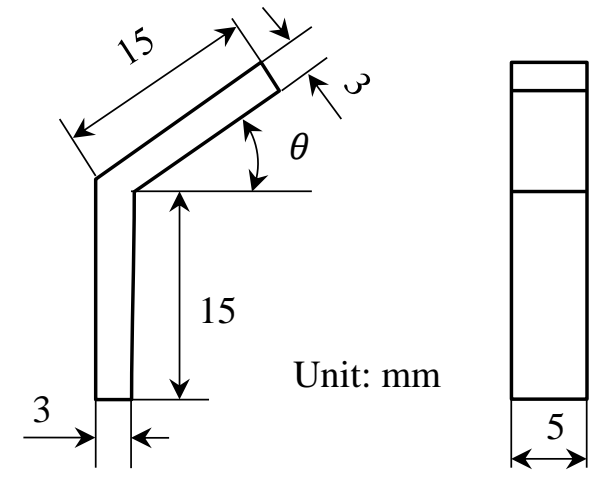

Fig 1. Experimental model

The FDM 3D printer is utilized for manufacturing the 3D model. Furthermore, PolyLactic Acid (PLA) is prepared as main source of material to build up the 3D printed model and support structure. It has $1.75 \mathrm{~mm}$ of original diameter, whereas the diameter deposit material is set $0.4 \mathrm{~mm}$ through to the nozzle. All variation of $3 \mathrm{D}$ model is printed into two kind 3D models setting, with support structure addition and without support structure addition. The general setting of 3D printer software for all 3D models follows Table 1. 
Table 1. General 3D model setting in the 3D printer software

\begin{tabular}{lc}
\hline \multicolumn{1}{c}{ Setting } & Value \\
\hline Layer Height & $0.15 \mathrm{~mm}$ \\
Wall thickness & $1 \mathrm{~mm}$ \\
Infill density & $10 \%$ \\
Infill pattern & Zig-zag \\
Printing temperature & $200^{\circ} \mathrm{C}$ \\
Build plate temperature & $60{ }^{\circ} \mathrm{C}$ \\
Printing speed & $60 \mathrm{~mm} / \mathrm{s}$ \\
\hline
\end{tabular}

In the next process, the weight of all 3D printed models is measured using analytic weight scale which has accuracy $10^{-4} \mathrm{~g}$. The weight and surface quality both of $3 \mathrm{D}$ printed models are compared and analyzed.

\section{Result and Discussions}

The surface quality of $3 \mathrm{D}$ printed model is main parameter that should be considered, especially on overhang surface. Not only the surface quality of 3D printed model, but also the weight of material usage is considered to find out the support structure addition effect. Furthermore, the material usage, overhang angle effect and surface quality of 3D printed model are main focus discussion on this study.

\section{A. The Supports Structure Addition Effect to Weight of 3D Printed Model}

The support structure has main function to prevent the main object structure from collapsing. Actually, the support usage is depended to orientation of $3 \mathrm{D}$ model. The different support orientations will also influence to the final printed mechanical properties [9]. By choosing the optimal part orientation, Paul and Anand [10] had analyzed the manufacturing of precision parts while additive manufacturing processes with a minimal number of support structures. Then Das et al. [11] identified a better build direction which can reduce the support material used for a direct metal laser sintering based process. In the general application, the support structure is built from base to the overhang shape (Fig. 2a). The 3D printer machine has a single source of material, so that the support structure material is also taken from it. The characteristic of support structure is easy to remove from main 3D printed model. In this study, sixteen of $3 \mathrm{D}$ printed models with support structure addition success to be printed for example on the $3 \mathrm{D}$ printed model with $0^{\circ}$ of overhang angle (Fig. 2b). The falling deposit layer does not visible on the overhang shape after removing the support material (Fig. 2b). 


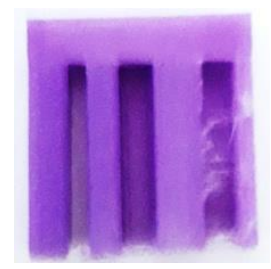

(a)

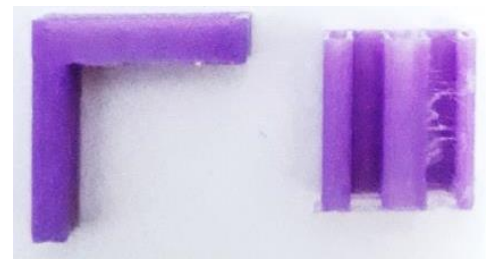

(b)

Fig 2. Experimental printed models a) with support structure b) removing support structure

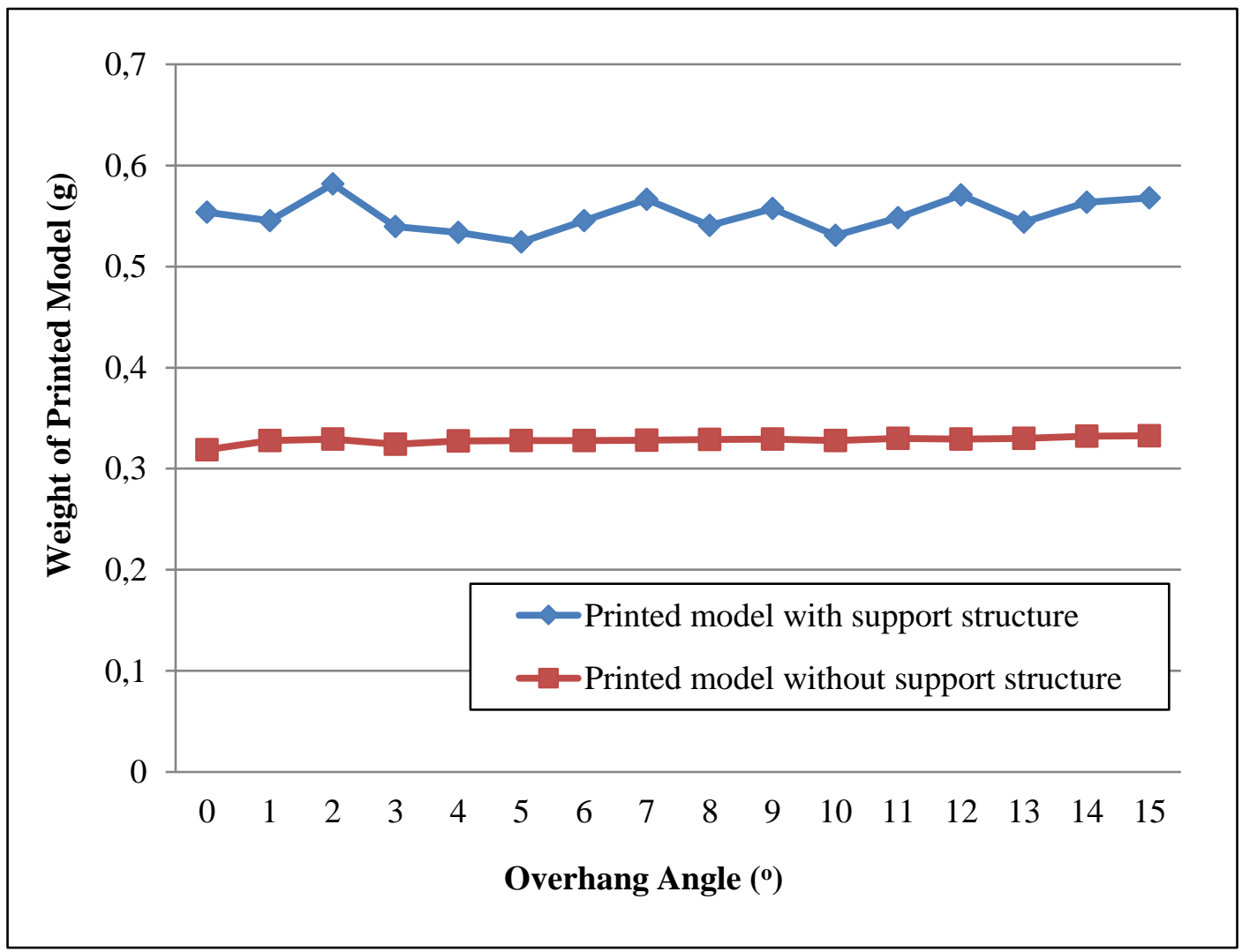

Fig 3. Weight of 3D printed material to build the both type 3D model

The support material usage can increase consume material as shows in the Fig. 3. The average weight of one 3D printed model with support structure addition is 0.5508 $\mathrm{g}$, otherwise the $3 \mathrm{D}$ printed model without support structure addition is $0.3282 \mathrm{~g}$. By using equation (1), where $\overline{W P M}_{\text {support }}$ is average weight of printed model with support structure addition, whereas $\overline{W P M}_{\text {non support }}$ is average weight of printed model without support structure addition, the 3D printed model with support structure addition has average overweight around $40.41 \%$ than without support structure addition. It indicates that the material usage is a lot and leans to waste the material. The support material should be optimized so as to minimize material usage [12]. However, as a manufacturing process, the producing and removing support material show the significant potential increases in material consumption, energy usage and the several of manual post- 
processing required to produces the final part [12]. As well as, this situation indicates that there is an increase in production costs.

$$
\text { Overweight }(\%)=\frac{\overline{W P M}_{\text {support }}-\overline{W P M}_{\text {non support }}}{\overline{W P M}_{\text {support }}} \times 100
$$

\section{B. The Overhang Angle Effect of 3D Model to Surface Quality of 3D Printed Model}

The support structure usage has optimized at $45^{\circ}, 60^{\circ}$ and $90^{\circ}$ of overhang angle 3D model [13]. In this study, the sixteen kind of overhang angle is set up in this experiment object to find out the minimum angle in term for support structure needed. All 3D printed models with support structure addition were successfully manufactured but it shows different result on 3D printed models without support structure addition. The falling deposit layer depicts on the 3D printed model (Fig. 4), especially on $0^{\circ}$ until $11^{\circ}$ of overhang angle. This phenomenon is caused by there is no support material which prevent the deposit layer. Whereas the printed model is successfully to be printed on remain variation model. It can occur when the early deposit structure capable to prevent the next deposit layer.

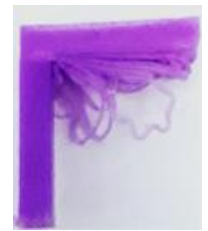

$\left(0^{\circ}\right)$

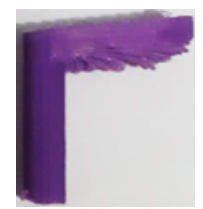

$\left(6^{\circ}\right)$

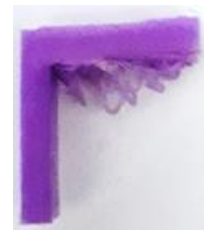

$\left(1^{\circ}\right)$

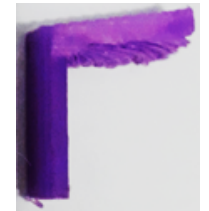

$\left(7^{\circ}\right)$

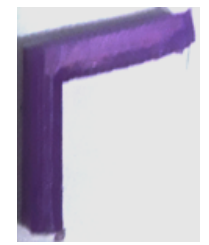

$\left(12^{\circ}\right)$

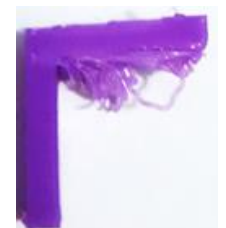

$\left(2^{\circ}\right)$

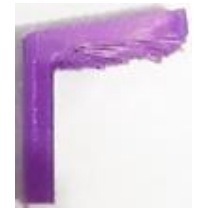

$\left(8^{\circ}\right)$

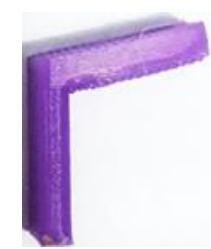

$\left(13^{\circ}\right)$

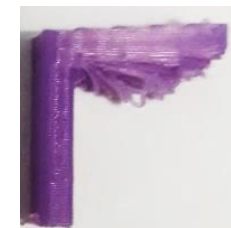

$\left(3^{\circ}\right)$

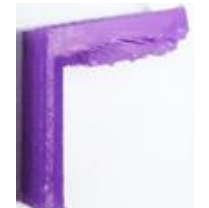

$\left(9^{\circ}\right)$

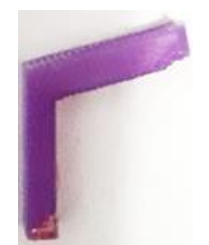

$\left(14^{\circ}\right)$

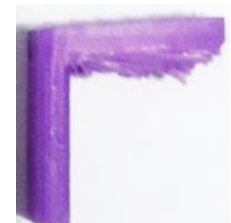

$\left(4^{\circ}\right)$

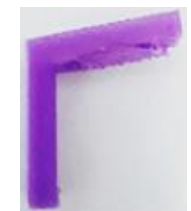

$\left(10^{\circ}\right)$

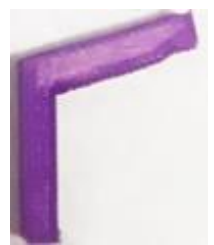

$\left(15^{\circ}\right)$

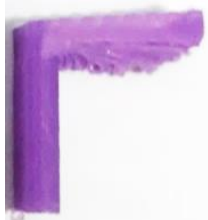

$\left(5^{\circ}\right)$

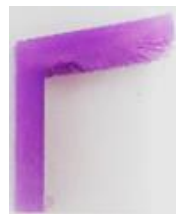

$\left(11^{\circ}\right)$

Fig 4. 3D printed model without support structure

The quality of printed model is also good enough, that meaning without any defect on the surface of overhang area. Its overhang surface quality is better than $3 \mathrm{D}$ printed model with support structure addition (Fig. 5) because the remaining of support material sometimes makes the overhang surface rough. 


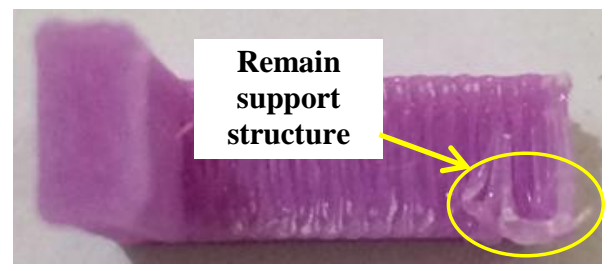

(a)

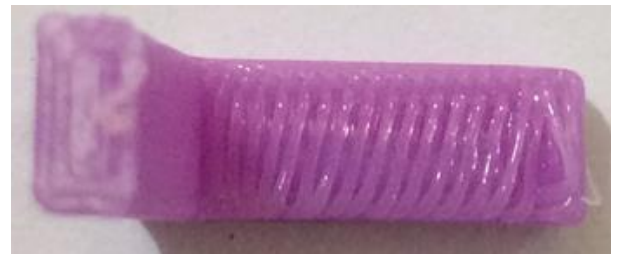

(b)

Fig 5. Quality surface of overhang area a) 3D printed with support structure and b) 3D printed without support structure

\section{Conclusion}

The 3D printing technology is one technology to product the physical 3D model layer by layer. The support structure is commonly included on the process for prevent the printed model from collapsing, especially for overhang shape. On this study, the support structure addition can be successfully printed for all 3D models but the material usage is $40.41 \%$ overweight than 3D printed model without support structure addition. Furthermore, the support structure can be built up but it does not need to generate if the overhang angle more than $11^{\circ}$.

\section{Acknowledgment}

I wish to thank for all members on this study for their valuable technical support on this project. My special grateful thanks are also extended to Ms. Rr. Poppy for permitting us to use the measurement device on the nano-material Laboratory.

\section{References}

[1] S. Bose, S. Vahabzadeh, and A. Bandyopadhyay, "Bone tissue engineering using 3D printing," Mater. Today, vol. 16, no.12, pp.496-504, 2013.

[2] S. Ford and T. Minshall, "Invited review article: Where and how 3D printing is used in teaching and education," Addit. Manuf., vol. 25, pp.131-150, 2019.

[3] X. Chen, L. Xu, Y. Wang, Y. Hao, and L. Wang, "Image-guided installation of 3D-printed patient-specific implant and its application in pelvic tumor resection and reconstruction surgery," Comput. Methods Programs Biomed., vol.125, pp.66-78, 2016.

[4] CH. V. Madhav, R. S. N. H. Kesav, Y. S. Narayan, "Importance and Utilization of 3D Printing in Various Applications," pp. 24-29, 2016.

[5] Y. Weng, M. Li, M. J. Tan, S. Qian, "Design 3D printing cementitious materials via Fuller Thompson theory and Marson-Percy model," Constr. Build. Mater., 163:600-610, 2018.

[6] P. Dudek and K. Zagórski, "Cost, resources, and energy efficiency of additive manufacturing," E3S Web Conf., vol. 14, pp. 1-8, 2017.

[7] A. T. Gaynor and J. K. Guest, "Topology optimization considering overhang constraints: Eliminating sacrificial support material in additive manufacturing through design," Struct. Multidiscip. Optim., vol. 54, no. 5, pp. 1157-1172, 2016. 
[8] Thomas. D, "The development of design rules for selective laser melting," University of Wales Institute, 2009.

[9] J. Cantrell, S. Rohde, D. Damiani, R. Gurnani, L. DiSandro, J. Anton, A. Young, A. Jerez, D. Steinbach, C. Krsoese, P. Ifju, "Experimental characterization of the mechanical properties of 3D-printed ABS and polycarbonate parts," Rapid Prototyp. J., vol. 23, pp. 811-824. 2017

[10] R. Paul, S. Anand, "Optimization of layered manufacturing process for reducing form errors with minimal support structures," J. Manuf. Syst., vol. 36, pp. 231243, 2015.

[11] P. Das, R. Chandran, R. Samant, S. Anand, "Optimum Part Build Orientation in Additive Manufacturing for Minimizing Part Errors and Support Structures," Procedia Manuf., vol. 1, pp. 343-354, 2015.

[12] J. Jiang and X. Xu, "Support Structures for Additive Manufacturing : A Review," J. Manuf. Mater. Process., vol. 2, pp. 1-23, 2018.

[13] A. Garaigordobil and R. Ansola, "A new overhang constraint for topology optimization of self-supporting structures in additive manufacturing," Structural and Multidisciplinary Optimization, vol. 58, no. 5, pp. 2003-2017, 2018. 